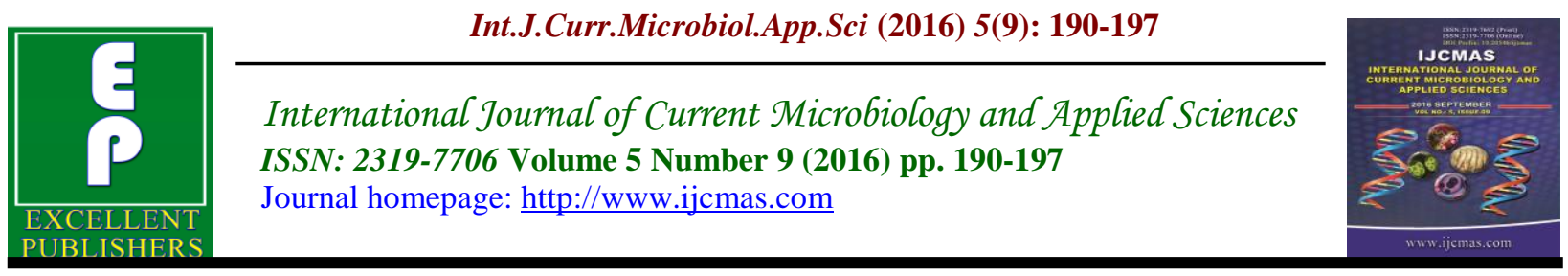

Original Research Article

http://dx.doi.org/10.20546/ijcmas.2016.509.021

\title{
Prevalence of Intestinal Parasites among Patients attending a Tertiary Care Centre in South India
}

\author{
Kumar Manochitra, Shashiraja Padukone, Selvaratthinam \\ Ajay Philips and Subhash Chandra Parija*
}

\begin{abstract}
Department of Microbiology, Jawaharlal Institute of Postgraduate Medical Education and Research (JIPMER), Puducherry, India

*Corresponding author
\end{abstract}

Keywords

Intestinal parasites,

Distribution,

Prevalence,

Protozoal infections,

Helminthic

infections, South

India, Entamoeba

spp., Blastocystis,

Ascaris.

Article Info

Accepted:

13 August 2016

Available Online:

10 September 2016
A B S T R A C T

Intestinal parasitic infections are important public health problem and are globally endemic. It constitutes single worldwide cause of morbidity and mortality. The prevalence is presumed to be high in developing countries like India probably due to poor sanitary conditions and improper personal hygiene. This study was estimate the burden of the intestinal parasitic infections and the current pattern of distribution in patients attending a tertiary care setting in Puducherry, India. This is a retrospective study carried out over a period of 5 years (Aug 2011 to Jun 2016). Stool samples of patients received in the parasitology section, Dept. of Microbiology, JIPMER were subjected to routine stool investigations. The results were recorded, and data was presented in the form of frequency and distribution. A total of 1508 fresh stool samples were screened, of which $22.2 \% \quad(n=335)$ were positive for various intestinal parasites. Overall, protozoal infections $(16.25 \%)$ were higher than helminthic infections (5.97\%). On the whole, Entamoeba species showed higher prevalence (39.7\%) followed by Blastocystis (13.4\%) and Ascaris (11.34\%). Among the coccidian parasites identified, Cystoisospora showed maximum positivity $(2.7 \%)$. There was a noticeable female predominance pattern (56\%) compared to male showing $43.9 \%$ positivity. The results conclude that Entamoeba spp., and Ascaris are the most common protozoal and helminthic parasites respectively present in the study population. In our study population, age group between 31-40 years are predominantly infected with these parasites. Apart from the early diagnosis and effective treatment, health education is a requisite to control infections in developing countries.

\section{Introduction}

Intestinal parasitic infections are believed to be the most common and important public health problem worldwide. These parasites constitute the greatest single universal cause of morbidity and mortality. More than $60 \%$ of the global population is infected with enteric parasites (WHO, 2002). The prevalence is said to be high in developing countries, probably due to poor sanitary conditions and improper personal hygiene. 
The other factors attributable to the prevalence of these infections are poverty, illiteracy, tropical hot and humid weather conditions and contaminated drinking water resources (Sadeghi and Borji, 2015). Consequently, the epidemiological pattern of these parasites varies in different geographical regions.

Parasitic infections cause substantial hindrance to socio-economic developments, particularly in developing countries. This can be due to the ill effects and retarded physical and mental development of children caused by the parasites. Approximately 39 disability-adjusted life years (DALY) is associated with enteric parasitic infections, thus representing a major global and economic burden (Ramana, 2012).

Enteric protozoan parasites and the soiltransmitted helminths are responsible for gastrointestinal disturbances leading to infections. The WHO report states that amoebiasis caused by the protozoan parasite Entamoeba histolytica is the third most common parasitic cause of morbidity and mortality, with an estimate of about 50 million infections worldwide (WHO, 1997) followed by giardiasis caused by Giardia intestinalis/ duodenalis and cryptosporidiosis caused by Cryptosporidium spp. Ascaris lumbricoides and Hymenolepsis nana are the commonest nematode and cestode affecting approximately 1 billion people (CDC, 2006).

Intestinal parasitic infections are a major source of health concern in India like in any other developing nations. The overall prevalence of intestinal infections, caused by enteric parasites ranges from $12.5-67 \%$ in our country. Limited studies determining the prevalence of intestinal parasitic infections have been published previously from South India (Gang et al., 1998; Fernandez et al., 2002; Dhanabal et al., 2014). These studies report the prevalence of intestinal parasitic infections among slum-dwellers and children of rural and urban locations of Chennai, Tamilnadu respectively (Dhanabal et al., 2014; Fernandez et al., 2002). The prevalence of the pathogenic intestinal parasites has been determined among a Southern Indian rural community from Vellore district of Tamilnadu (Gang et al., 1998).

Studies related to the overall frequency of enteric parasites in the Union Territory of Puducherry have been scanty (Parija \& Rao, 1987); whereas, a study related specifically to helminthic infections in school children from Puducherry have been published in the recent past (Ragunathan et al., 2010). To our knowledge, no recent studies, taking into account the overall prevalence of the intestinal parasites from Puducherry has been reported. Therefore, this study was conducted to determine the present distribution pattern of the intestinal parasites among patients attending a tertiary care hospital in Puducherry, South India.

\section{Methodology}

\section{Study design}

This is a hospital-based retrospective study for a period of five years (2011- 2016) conducted in the Parasitology Section, Department of Microbiology, JIPMER, Puducherry, India. Patients in all age groups, both the genders with or without gastrointestinal disturbance were included in this study. The approval to use the samples for this study was obtained from JIPMER Institute Ethics Committee (IEC). Stool samples of patients received in the section were subjected to routine stool investigation 
during the study period. The macroscopic examination was carried out for the presence of helminthic body segments and to record consistency of stool. Further, various microscopic examination methods such as direct wet mount, stool concentration techniques and staining methods (Wheatley's modified Trichrome staining and modified acid-fast staining) were employed for efficient detection of the parasites. The results were recorded, and the data was analysed in the form of frequency and distribution.

\section{Results and Discussion}

A total of 1508 samples were screened, of which $22.21 \% \quad(n=335)$ were positive for intestinal parasites. In general, protozoal infections (16.25\%) showed a higher prevalence pattern compared helminthic infections (5.97\%) (Figure: 1A). During the study period of approximately 5 years, enteric protozoan parasites (Entamoeba histolytical E. disparl E. moshkovskii, Entamoeba coli, Giardia intestinalis/ duodenalis, Blastocystis and Balantidium coli), coccidian parasites (Cystoisospora, Cyclospora and Cryptosporidium spp.,) and helminths (Ascaris, Trichuris trichura, Enterobius and Taenia) were identified in our study population.

Among the parasites identified, Entamoeba spp., (39.7\%) was found to be the commonest, followed by Blastocystis (13.4\%) and Ascaris (11.34\%). Among the coccidian parasites, Cystoisospora showed maximum positivity $(2.7 \%)$ preceded by Cryptosporidium spp., (1.5\%). Other uncommon parasites encountered were Taenia egg (1.19\%), Balantidium coli (0.6\%) Trichuris trichura (0.3\%) and Enterobius egg (0.3\%) (Figure: 1B). With respect to the gender of the study population, there was a noticeable female predominance pattern $(56 \%)$ compared to male showing $43.92 \%$ positivity.

In this study, $22.2 \%$ of samples showed the presence of one parasite, $1.46 \%$ of the samples showed the presence of more than one parasite and $77.8 \%$ of the samples studied showed no parasites (Figure 2). The parasites that were frequently observed in co-infections were Entamoeba spp., Entamoeba coli and Blastocystis.

It was also observed that among the patients with parasitic infection, $52.5 \%$ of them showed clinical symptoms such as diarrhoea or dysentery with or without fever, colitis, ulcerative colitis, abdominal pain, etc., correlating to the infection with intestinal parasites. Approximately $47.4 \%$ of the patients found to be infected with one or more parasites showed no relevant clinical symptoms or they were said to be asymptomatic.

Intestinal parasites predominates the parasitic infection manifested in human. It is an important public health problem worldwide predominantly in tropics, subtropics and resource-poor settings. Prevalence based studies will aid in conceptualising the spread of these parasites in a better way. In the current study, we report an overall prevalence of $22.21 \%$ with prevalence rates differing for individual parasites. It is known that the overall prevalence of these parasites, in India ranges from $12.5-67 \%$ as reported previously (Gang et al., 1998).

On the whole, this study reported a higher prevalence of Entamoeba spp., (39.7\%) that was consistent with the previous reports from Puducherry and South India (Parija \& Rao, 1987; Fernandez et al., 2002; Dhanabal et al., 2014). Amoebiasis caused by $E$. histolytica is the third common parasitic 
cause of morbidity and mortality worldwide. Global prevalence of Entamoeba spp., ranges from 2- $60 \%$ whereas in the Indian scenario, it is $3.6-47.4 \%$ (Khairnar \& Parija, 2002). Previous study apropos the prevalence of parasitic infection in Puducherry region conducted by Parija \& Sambasiva Rao in 1987 reported $E$. histolytica $(15.06 \%)$ to be the prevalent protozoal infection; however according to this finding, the most common parasite in that period was Ascaris (Parija \& Rao, 1987). The study conducted by Fernandez et al., among children in rural and urban settings of Chennai, also illustrated a higher prevalence of Entamoeba spp., (44.9\%); whereas, study by Dhanabal et al., in the low socioeconomic areas in South Chennai, India showed comparatively lesser prevalence of Entamoeba spp.,(21.8\%) (Fernandez et al., 2002; Dhanabal et al., 2014).

Moreover, Entamoeba spp., shows a pattern of high prevalence in large cities particularly in the coastal region (Gang et al., 1998). Accordingly, the Union territory of Puducherry located in the coastal region of South India also shows a similar pattern of Entamoeba spp., distribution. The transmission of Entamoeba spp., is through faecal-oral route. Therefore the reason for the higher prevalence of this parasite may be due to contamination of either food or water resources.

The second most common protozoan parasite, as recorded by this study was Blastocystis (13.4\%). Blastocystis is cosmopolitan in distribution and it is one of the most frequent enteric parasites found in human stool samples in developing countries (Parija \& Jeremiah, 2013). The worldwide prevalence of Blastocystis ranges from 0.5- 62\% (Clark et al., 2013). In India, a molecular-based study from a healthy population reported $27 \%$ prevalence of Blastocystis (Pandey et al., 2015). However, the studies based on microscopic techniques have reported lesser prevalence rate ranging from 3-8\% (Prasad et al., 2000; Mohandas et al., 2002). In the present study, Blastocystis also observed in co-infection with Entamoeba spp., Of the total 32 samples were multiple parasitism was recorded, a maximum number of samples showed the presence of both Blastocystis and Entamoeba spp., (Figure 2).

Giardia spp., considered to be the most common parasite causing water-borne diarrhoea, was found to be the third common protozoan parasite prevalent in the locality of Puducherry. Globally, the prevalence of Giardia spp., ranged from 20-30\% (Sadeghi $\&$ Borji, 2015) and in India it ranges from 3.8 to $23.5 \%$ (Gang et al., 1998). In our study population, Giardia spp., showed a distribution frequency of about $7.8 \%$ followed by Entamoeba coli (7.1\%). Similar distribution pattern of Giardia spp., has been reported by different researchers, elsewhere from India (Rao et al., 1977; Ramesh et al., 1991). Apart from these common intestinal parasites infecting the human, we have also reported a case of a rare parasitic infection with Balantidium coli (Kumar et al., 2016).

Moving to the helminthic infections, in our study, the prevalence of Ascaris was $11.34 \%$ followed by Hookworm (8.7\%), Strongyloides stercolaris (5\%), Taenia (1.2\%), Trichuris trichura and Enterobius (each $0.3 \%$ ). The global ranking of soiltransmitted helminths (STHs) by WHO, states that Ascaris is the most frequent parasite followed by Hookworm and $T$. trichura (http://www.who.int/intestinal_ worms/en/). Considering the prevalence of Ascaris and Hookworm our results were on par with the global ranking, except for $T$. trichura which showed very low prevalence 
rate in our study population. Whereas $S$. stercolaris, the fifth major STH, recorded considerably higher prevalence among the samples analysed for this study, compared to T. trichura.

In this study, females showed a prevalence rate of $56 \%$ for intestinal parasites compared to males $(43.9 \%)$. Females harbouring larger proportion of parasites than males have been observed in similar studies (Dhanabal et al., 2014). Despite the fact that, the risk of intestinal parasitic infection does not depend on the gender, our study showed a notable predominance pattern in females, that cannot be ignored.

The age-specific prevalence profile of our study population suggests that adults in the age group of 31-40 years were mostly infected with intestinal parasites followed by children of 1-10 years. We could also notice that in patients without any relevant clinical symptoms, the prevalence of enteric parasites was $47.4 \%$. This result probably suggests that there could be a relatively higher number of asymptomatic carriers in this study location that has to be considered for community-based studies. Nutritional grade and immune level of the host determines whether the presence of a pathogenic parasite results in a clinical disease or not.

A notable difference was observed in the distribution pattern of helminths when the present study was compared with the previous study from the same setting by Parija and Sambasiva Rao. Entamoeba spp., was the most common protozoan parasite during both the time- periods with approximately 30 years gap between the studies, but the rate of prevalence has drastically increased from $15.06 \%$ in 1987 to $39.7 \%$ at present. Hookworm was more prevalent during the 1980's (Parija \& Rao, 1987); whereas Ascaris has shown higher prevalence in the current study.

Fig.1a Frequency of protozoan, coccidian and helminthic parasites

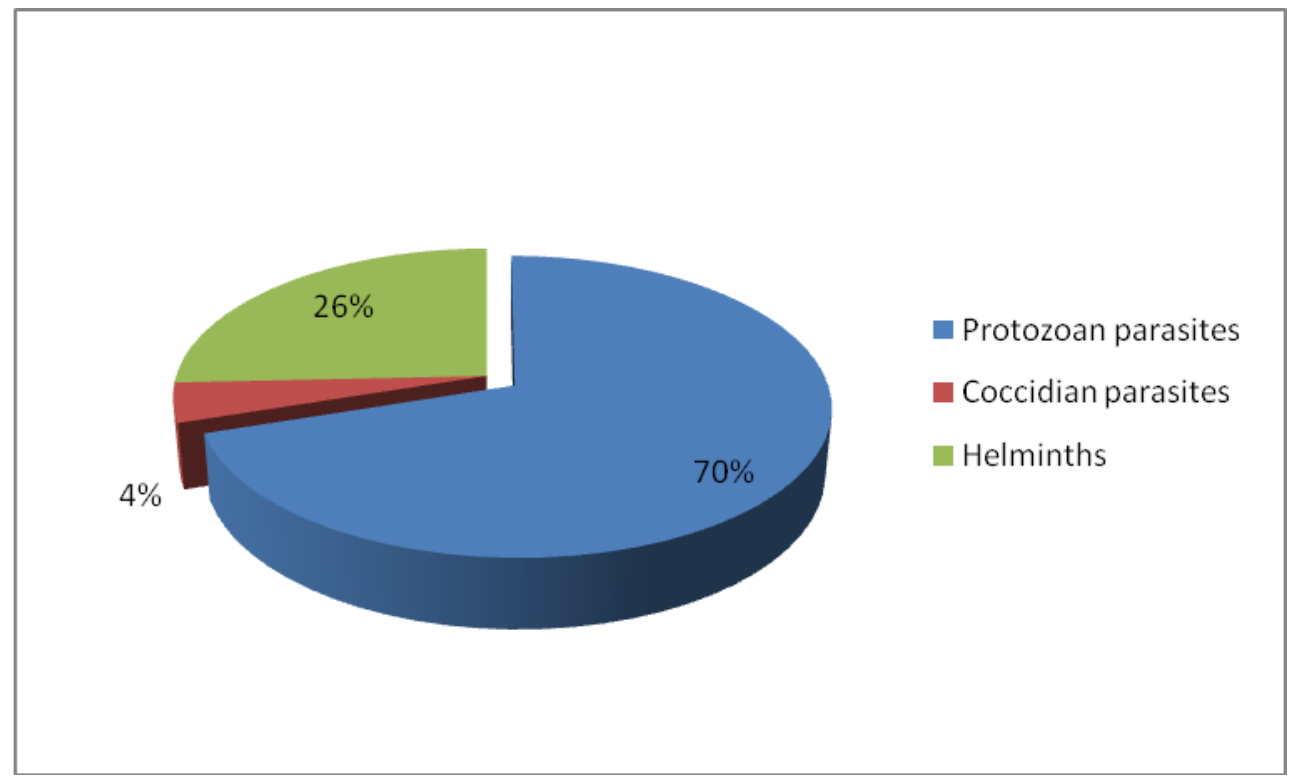

Fig.1b Distribution of intestinal parasites in the study population 


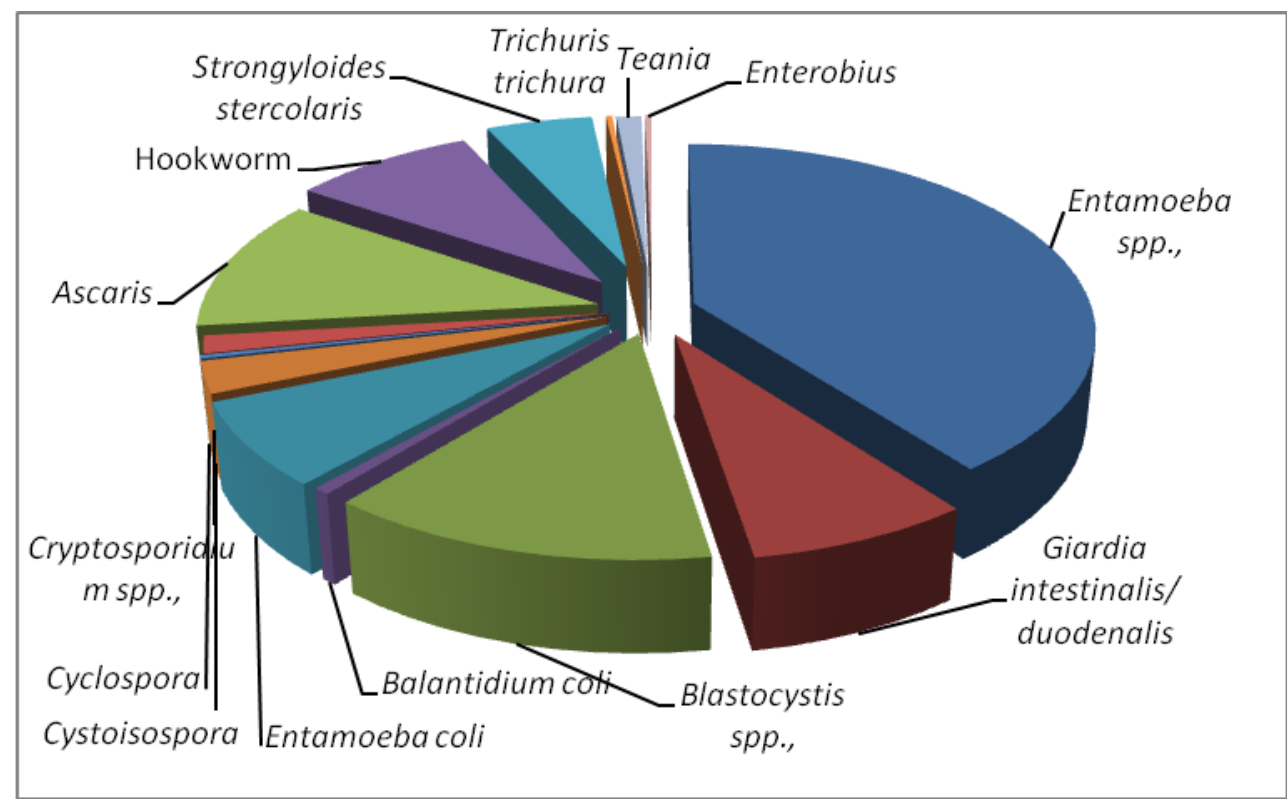

Fig.2 Parasites occurring as co-infections and total number identified in our study population

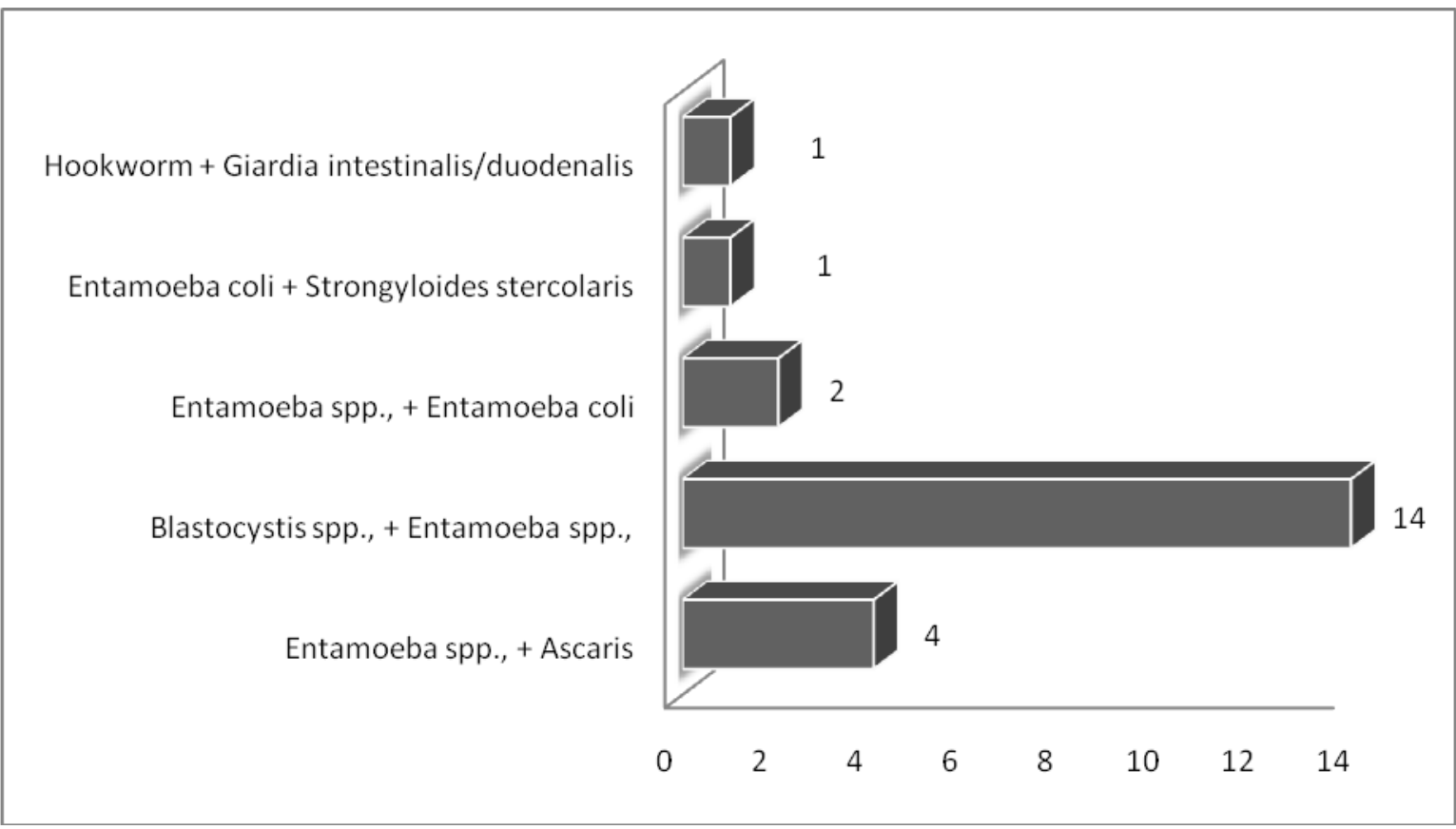

The limitation of the current study was that examination of a single stool specimen from each participant is not adequate and the methods were not very sensitive. Triple faeces test could have increased the frequency of the parasitic occurrence to some folds compared to that of a single sample. Regarding specificity, the true prevalence of the pathogenic E. histolytica and the other look-alike species (E. dispar and E. moshkovskii) and sub-type analysis for Blastocystis could not be determined with the conventional detection methods. For this purpose, molecular analysis based 
on nested-multiplex PCR assay has been performed for the samples and the data generated is currently being analysed for sensitivity and specificity. This study did not involve any data related to treatment. Our future perspective is to incorporate treatment options and follow-up after treatment, to determine its efficacy.

To conclude, this study sheds light on the current pattern of distribution of intestinal parasites, its burden on the health-care system and its deteriorating effect on the society as a whole. This study and similar previous studies suggest that India remains an endemic region for most of these enteric parasitic infections. Understanding the burden of these enteric parasites substantiates the need to determine their public health significance and to devise proper control measures. Apart from early diagnosis and effective treatment, health education, better sanitary management and evidence-based approaches are a prerequisite to control infections in developing and under-developed nations.

\section{Grant Disclosures}

This study was supported by Indian Council of Medical Research (ICMR), Govt. of India grant no. 5/8-1(27)2011-12ECD-II. The first author $(\mathrm{KM})$ is grateful to Council of scientific and industrial research (CSIR), Govt. of India, for providing financial support (grant no. 09/05(0007)/2012-EMRI). The study was also partly supported by JIPMER Institute Research Council Intramural Grant.

\section{Acknowledgement}

The authors would like to thank Ms. Karthika and Ms. Indumathy, Lab assistants, for their immense help in data collection and maintenance of records.

\section{References}

CDC, DPDx. 2006. Laboratory identification of parasite of Public Health Concern. Atlanta: Centre for Disease Control \& Prevention, USA.

Clark, C.G., van der Giezen, M., Alfellani, M.A., Stensvold, C.R. 2013. Recent developments in Blastocystis research. Adv. Parasitol., 82: 1-32

Dhanabal, J., Selvadoss, P.P., Muthuswamy, K. 2014. Comparative Study of the Prevalence of Intestinal Parasites in Low Socioeconomic Areas from South Chennai, India. J. Parasitol. Res., 1-8.

Fernandez, M.C., Verghese, S., Bhuvaneswari, R., Elizabeth, S.J., Mathew, T., Anitha, A., Chitra, A.K. 2002. A comparative study of the intestinal parasites prevalent among children living in rural and urban settings in and around Chennai. $J$. Comm. Dis., 34: 35-39.

Kang, G., Mathew, M.S., Rajan, D.P., Daniel, J.D., Mathan, M.M., Mathan, V.I., Muliyil, J.P. 1998. Prevalence of Intestinal parasites in rural Southern Indians. Trop. Med. Int. Health, 3: 7075.

Khairnar, K., Parija, S.C. 2002. A novel nested multiplex PCR assay for differential detection of Entamoeba histolytica, E. moshkovskii and E. dispar DNA in stool samples. BMC Microbiol., 7: 47.

Kumar, M., Rajkumari, N., Mandal, J., Parija, S.C. 2016. A case report of an uncommon parasitic infection of human balantidiasis. Trop Parasitol., 6: 82-4.

Mohandas, Sehgal, R., Sud, A., Malla, N. 2002. Prevalence of intestinal parasitic pathogens in HIV-seropositive individuals in Northern India. Jpn. J. Infect. Dis., 55: 83-4. 
Pandey, P.K., Verma, P., Marathe, N., Shetty, S., Bavdekar, A., Patole, M.S., Stensvold, C.R., Shouche, Y.S. 2015. Prevalence and subtype analysis of Blastocystis in healthy Indian individuals, Infect. Genet. Evol., 31: 296-299.

Parija, S.C., Jeremiah, S. 2013. Blastocystis: Taxonomy, biology and virulence. Trop. Parasitol., 3: 17-25.

Parija, S.C., Rao, R.S. 1987. Prevalence of parasitic infections in Pondicherry. Indian J. Parasitol., 11: 63-65.

Prasad, K.N., Nag, V.L., Dhole, T.N., Ayyagari, A. 2000. Identification of enteric pathogens in HIV-positive patients with diarrhoea in northern India. J. Health Popul. Nutr., 8: 23-6.

Ragunathan, L., Kalivaradhan, S.K., Ramadass, S., Nagaraj, M., Ramesh, K. 2010. Helminthic Infections in School Children in Puducherry, South India. J. Microbiol. Immunol. Infect., 43: 228-232.
Ramana, K.V. 2012. Intestinal parasitic infections: An overview. Ann. Trop. Med. Public Health, 5: 279-281.

Ramesh, G.N., Malla, N., Raju, G.S., Sehgal, R., Ganduly, N.K., Mahajan, R.C., Dilawari, J.B. 1991. Epidemiological study of parasitic infestations in lower socio-economic group in Chandigarh (North India), Indian J. Med. Res., 93: 47-50.

Rao, C.K., Krishnaswami, A.K., Biswas, H. 1977. Prevalence of intestinal parasites in selected village of Mahasu district, Himachal Pradesh. Indian J. Med. Res., 56: 1959-65.

Sadeghi, H., Borji, H. 2015. A survey of intestinal parasites in a population in Qazvin, north of Iran. Asian Pac. J. Trop. Dis., 5: 231-233.

World Health Organization. 1997. "Amoebiasis", WHO Weekly Epidemiol. Record, 72: 97-100.

World health Organization. 2002. Prevention and control of Intestinal parasitic infections. Geneva: WHO.

\section{How to cite this article:}

Kumar Manochitra, Shashiraja Padukone, Selvaratthinam, Ajay Philips and Subhash Chandra Parija. 2016. Prevalence of Intestinal Parasites among Patients attending a Tertiary Care Centre in South India. Int.J.Curr.Microbiol.App.Sci. 5(9): 190-197. doi: http://dx.doi.org/10.20546/ijcmas.2016.509.021 